\title{
A COMPACT QUALITATIVE UNCERTAINTY PRINCIPLE FOR SOME NONUNIMODULAR GROUPS
}

\author{
WASSIM NASSERDDINE
}

(Received 21 April 2018; first published online 28 November 2018)

\begin{abstract}
Let $G$ be a separable locally compact group with type $I$ left regular representation, $\widehat{G}$ its dual, $A(G)$ its Fourier algebra and $f \in A(G)$ with compact support. If $G=\mathbb{R}$ and the Fourier transform of $f$ is compactly supported, then, by a classical Paley-Wiener theorem, $f=0$. There are extensions of this theorem for abelian and some unimodular groups. In this paper, we prove that if $G$ has no (nonempty) open compact subsets, $\hat{f}$, the regularised Fourier cotransform of $f$, is compactly supported and $\operatorname{Im} \hat{f}$ is finite dimensional, then $f=0$. In connection with this result, we characterise locally compact abelian groups whose identity components are noncompact.
\end{abstract}

2010 Mathematics subject classification: primary 43A30; secondary 43A25.

Keywords and phrases: Paley-Wiener property, qualitative uncertainty principle, Fourier algebra, Fourier transformation, Fourier cotransformation.

\section{Introduction}

Let $f \in L^{1} \cap L^{2}(\mathbb{R})$. Then, by a classical Paley-Wiener theorem, $f$ is compactly supported if and only if its Fourier transform $\hat{f}$ extends to $\mathbb{C}$ as an entire function of exponential type. A consequence of this result is the following property: if $f$ has compact support, then $\hat{f}$ cannot have compact support unless $f=0$. This property, which holds on $\mathbb{R}$, fails on an arbitrary locally compact abelian group. In this paper, we seek an analogue of this property for locally compact groups and characterise the groups that satisfy this property.

Let $G$ be a separable locally compact group, $\widehat{G}$ its dual and $A(G)$ its Fourier algebra. If $G$ is abelian and if $f \in A(G)$ (or $f \in L^{1}(G)$ ) has compact support and its Fourier transform $\hat{f}$ has compact support, then $f=0$. We call this the compact qualitative uncertainty principle (CQUP) for $G$. It is a version of the classical qualitative uncertainty principle that a function and its Fourier transform cannot both be too

This project has been funded with support from the Lebanese University.

This is an Open Access article, distributed under the terms of the Creative Commons Attribution licence (http://creativecommons.org/licenses/by/4.0/), which permits unrestricted re-use, distribution, and reproduction in any medium, provided the original work is properly cited.

(C) 2018 Australian Mathematical Publishing Association Inc. 
localised unless the function is identically zero. For other versions of the qualitative uncertainty principle on unimodular and Moore groups, see [1, 7, 8, 10] and, for a topological Paley-Wiener property involving the reduced dual of a locally compact group, see [9].

The key results of harmonic analysis on abelian and unimodular groups (such as the Plancherel formula [11, page 213] and the Fourier inversion formula [11, page 221]) are realised by the Fourier transform. Indeed, if $G$ is unimodular of type $I$, then the Fourier transform $\mathcal{F}(f)$ of a function $f \in A(G) \cap L^{1}(G)$ forms a field of bounded operators $\mathcal{F}(f)=(\pi(f))_{\pi \in \widehat{G}}$ on $\widehat{G}$ with $\mathcal{F}(f) \in L^{1}(\widehat{G})$, and $f$ is recovered from its Fourier transform by $f(x)=\int_{\widehat{G}} \operatorname{Tr}\left[\mathcal{F}(f)(\pi) \pi(x)^{-1}\right] d_{\mu}(\pi)$, where $\mu$ is the (unique) Plancherel measure on $\widehat{G}$ associated with a fixed Haar measure $v$ on $G$ [11, Corollary 4.3, page 223]. However, if the group is not unimodular, the Fourier transform fails to give such results in the ordinary way and must be tempered by unbounded operators. If $G$ (is nonunimodular and) has type $I$ left regular representation, the Fourier inversion theorem [14, Theorem 4.5] states that a function $f \in A(G) \cap L^{1}(G)$ is recovered from its regularised Fourier cotransform $\mathcal{F}(f) \circ \mathcal{K}$ by means of $f(x)=\int_{\widehat{G}} \operatorname{Tr}\left[\mathcal{F}(f)(\pi) K_{\pi} \pi(x)^{-1}\right] d_{\mu}(\pi)$, where $\mu$ is a Plancherel measure, unique up to equivalence, on $\widehat{G}$ and $\mathcal{K}=\left(K_{\pi}\right)_{\pi \in \widehat{G}}$ is a specific measurable field of (unbounded) positive self-adjoint operators. If $G$ is unimodular, then $\mathcal{K}=\left(\operatorname{Id}_{H_{\pi}}\right)_{\pi \in \widehat{G}}$ and thus $\hat{f}=\mathcal{F}(f)$. As a consequence, it is natural, in our approach to the CQUP, to deal with the regularised Fourier cotransformation as an extension of the Fourier transform.

In what follows, $G$ denotes a separable locally compact group with type $I$ left regular representation and $\hat{f}$ denotes the regularised Fourier cotransform of $f \in A(G)$ [13, page 547]. For $\pi \in \widehat{G}$, we denote the associated representation space by $\mathcal{H}_{\pi}$. Let $d \in \mathbb{N}^{*}, M_{\pi}$ be a subspace of $\mathcal{H}_{\pi}$ of dimension $d_{\pi} \leq d$ and $M:=\int_{\widehat{G}}^{\oplus} M_{\pi} d \mu(\pi)$. Let $K$ and $\widehat{H}$ be compact subsets of $G$ and $\widehat{G}$, respectively. Write supp $\hat{f} \subseteq M_{\widehat{H}}$ if $\operatorname{Im} \hat{f}(\pi) \subseteq M_{\pi}$ for every $\pi \in \widehat{G}$ and $\hat{f}$ vanishes outside $\widehat{H}$. Let

$$
A_{K, M_{\overparen{H}}}(G)=\left\{f \in A(G): \operatorname{supp} f \subseteq K \text { and } \operatorname{supp} \hat{f} \subseteq M_{\widehat{H}}\right\} .
$$

We say that the CQUP is valid on $G$ if $A_{K, M_{\overparen{H}}}(G)=\{0\}$ for all $d, K, \widehat{H}$ as above. We will prove that $A_{K, M_{\overparen{H}}}(G)$ is finite dimensional and, if $G$ has no (nonempty) open compact subsets, then the CQUP holds on $G$. These results generalise their analogues [12, Theorem 4 and Corollary 5, page 495] on the affine group of a local field and give a new characterisation of locally compact abelian groups whose identity components are noncompact. Suppose that $G$ is abelian and let $f \in L^{1}(G), A_{f}=\{x \in G: f(x) \neq 0\}$ and $B_{f}=\{\hat{x} \in \widehat{G}: \hat{f}(\hat{x}) \neq 0\}$. As in [5], the group $G$ is said to satisfy the qualitative uncertainty principle (QUP) if, for every $f \in L^{1}(G)$,

$$
v\left(A_{f}\right)<v(G) \text { and } \mu\left(B_{f}\right)<\mu(\widehat{G}) \Rightarrow f=0 \text { a.e. }
$$

A consequence of our results is that the CQUP and the QUP are equivalent on noncompact abelian groups. 


\section{Results}

Throughout this paper, $G$ denotes a separable locally compact group with type $I$ left regular representation equipped with a left Haar measure $v$. Let $\Delta$ be the modular function of $G$ and $A(G)$ the Fourier algebra of $G$. For a complex function $f$ on $G$, we adopt the notation: $\check{f}(x)=f\left(x^{-1}\right), \tilde{f}(x)=\overline{f\left(x^{-1}\right)}$. Let $\widehat{G}$ be the dual of $G$. Then there are a standard measure $\mu$ on $\widehat{G}$, which we call the Plancherel measure (see [2, page 225] or [13, page 545]), a $\mu$-measurable field $\left(\pi, \mathcal{H}_{\pi}\right)_{\pi \in \widehat{G}}$ of representations of $G$ and a measurable field $\mathcal{K}=\left(K_{\pi}\right)_{\pi \in \widehat{G}}$ of nonzero positive self-adjoint operators such that, for almost all $\pi \in \widehat{G}, K_{\pi}$ is semi-invariant with weight $\Delta^{-1}$, the operator $\pi(f) K_{\pi}^{1 / 2}$, where $f \in L^{1} \cap L^{2}(G)$, extends to a Hilbert-Schmidt operator on $\mathcal{H}_{\pi}$, denoted $\mathcal{P}(f)(\pi)$, and the map $\mathcal{P}: L^{1} \cap L^{2}(G) \longrightarrow L^{2}(\widehat{G})$ extends uniquely to a unitary map of $L^{2}(G)$ onto $L^{2}(\widehat{G})$. In this case, the Plancherel formula, for $f \in L^{1} \cap L^{2}(G)$, is

$$
\int_{G}|f(x)|^{2} d x=\int_{\widehat{G}}\left\|\pi(f) \circ K_{\pi}^{1 / 2}\right\|_{2}^{2} d \mu(\pi) .
$$

We fix a Plancherel measure $\mu$ on $\widehat{G}$ and a measurable field of nonzero positive selfadjoint operators $\mathcal{K}=\left(K_{\pi}\right)_{\pi \in \widehat{G}}$ so that the conditions (i) and (ii) of [2, Theorem 5, page 225] hold. This measure is unique up to equivalence and, for almost all $\pi$, each $K_{\pi}$ is unique up to a constant multiple. Now, since $\mu$ is fixed, the field $\mathcal{K}=\left(K_{\pi}\right)_{\pi \in \widehat{G}}$ is unique. Following the case of the affine group of a local field (see [3, page 235]), the Fourier cotransformation $\overline{\mathcal{F}}$ is defined by

$$
\overline{\mathcal{F}}(T)(x)=\int_{\widehat{G}} \operatorname{Tr}[T(\pi) \pi(x)] d \mu(\pi),
$$

where $T \in L^{1}(\widehat{G})$ and $x \in G$. The regularised Fourier cotransform of a function $f \in A(G)$ is defined by $\hat{f}:=\overline{\mathcal{F}}^{-1}(\breve{f})$. By the remark below, this is equal to $\mathcal{F}(f) \circ \mathcal{K}$ if $f \in L^{1} \cap A(G)$, where $\mathcal{F}(f)$ is the Fourier transform of $f$. For further details and notation not explained here, see [13].

Lemma 2.1. Let $f \in L^{1}(G)$ with compact support. Then, for almost all $\pi \in \widehat{G}$,

$$
\pi(\Delta f) K_{\pi}=K_{\pi} \pi(f) .
$$

Proof. As in [14, Lemma 4.2], $\pi\left(\Delta^{1 / 2} f\right)\left(K_{\pi}\right)^{1 / 2}=\left(K_{\pi}\right)^{1 / 2} \pi(f)$. It follows that

$$
K_{\pi} \pi(f)=\left(K_{\pi}\right)^{1 / 2}\left(K_{\pi}\right)^{1 / 2} \pi(f)=\left(K_{\pi}\right)^{1 / 2} \pi\left(\Delta^{1 / 2} f\right)\left(K_{\pi}\right)^{1 / 2}=\pi(\Delta f) K_{\pi} .
$$

Theorem 2.2 (Inversion theorem [14, Theorem 4.5]). Suppose that $f \in A(G)$ and let $\mathcal{K}=\left(K_{\pi}\right)_{\pi \in \widehat{G}}$ be the field defined above. If $f \in L^{1}(G)$, then $\mathcal{F}(f) \circ \mathcal{K} \in L^{1}(\widehat{G})$, where $\mathcal{F}(f)$ is the Fourier transform of $f$, and

$$
f(x)=\int_{\widehat{G}} \operatorname{Tr}\left[\pi\left(x^{-1}\right) \mathcal{F}(f)(\pi) \circ K_{\pi}\right] d_{\mu}(\pi) .
$$


REMARK 2.3. In the abelian and some unimodular cases, the Fourier inversion theorem states that, for a class of functions, it is possible to recover a function from its Fourier transform. In our case $G$ is not unimodular and a function $f \in L^{1}(G) \cap A(G)$ cannot be recovered from its Fourier transform, but from its regularised Fourier cotransform $\hat{f}=\mathcal{F}(f) \circ \mathcal{K}$. Indeed, since $\hat{f}=\overline{\mathcal{F}}^{-1}(\check{f})$ and $\overline{\mathcal{F}}$ is bijective (by [13, Theorem 3.4, page 547]), $\overline{\mathcal{F}}(\hat{f})=\check{f}$ and hence

$$
f(x)=\check{f}\left(x^{-1}\right)=\overline{\mathcal{F}}(\hat{f})\left(x^{-1}\right)=\int_{\widehat{G}} \operatorname{Tr}\left[\hat{f}(\pi) \pi(x)^{-1}\right] d \mu(\pi) .
$$

TheOREM 2.4. Let $K$ be a compact subset of $G, \widehat{H}$ a compact subset of $\widehat{G}, d \in \mathbb{N}^{*}$ and, for each $\pi \in \widehat{G}$, let $M_{\pi}$ be a subspace of $\mathcal{H}_{\pi}$ of dimension $d_{\pi} \leq d$. Then the space

$$
A_{K, M_{\overparen{H}}}(G)=\left\{f \in A(G): \operatorname{supp} f \subseteq K \text { and } \operatorname{supp} \hat{f} \subseteq M_{\widehat{H}}\right\}
$$

is finite dimensional.

Proof. Without loss of generality, we can assume that $\mathcal{H}_{\pi}$ is infinite dimensional. Choose an orthonormal basis $\left\{e_{k}^{\pi}\right\}_{k=1, \ldots, d_{\pi}}$ of $M_{\pi}$ and complete it with an orthonormal basis of $M_{\pi}^{\perp}$, the orthogonal complement of $M_{\pi}$, to obtain an orthonormal basis $\left\{e_{k}^{\pi}\right\}_{k=1, \ldots, \infty}$ of $\mathcal{H}_{\pi}$. Then $M_{\pi}^{\perp} \subseteq \operatorname{Ker}(\hat{f}(\pi))^{*}$ for all $f \in A_{K, M_{\overparen{H}}}(G)$ and for all $\pi \in \widehat{G}$. Let us show that the ball

$$
B_{K, M_{\overparen{H}}}(G):=\left\{f \in A_{K, M_{\widehat{H}}}(G):\|f\|_{K} \leq 1\right\},
$$

where $\|f\|_{K}=\|f\|_{\infty}$ is the uniform norm of $f$ on $K$, is equicontinuous. Indeed, for all $f \in B_{K, M_{\overparen{H}}}(G)$ and $x$ in a neighbourhood of $x_{0}$ in $K$,

$$
\begin{aligned}
\left|f(x)-f\left(x_{0}\right)\right| & =\left|\int_{\widehat{H}} \operatorname{Tr}\left(\hat{f}(\pi)\left(\pi(x)^{-1}-\pi\left(x_{0}\right)^{-1}\right)\right) d \mu(\pi)\right| \\
& =\left|\int_{\widehat{H}} \sum_{k=1}^{d_{\pi}}\left\langle\left(\pi(x)^{-1}-\pi\left(x_{0}\right)^{-1}\right) e_{k}^{\pi},(\hat{f}(\pi))^{*} e_{k}^{\pi}\right\rangle d \mu(\pi)\right| \\
& \leq \mu(\widehat{H}) \sup _{\pi \in \widehat{H}} \sum_{k=1}^{d_{\pi}}\left\|\left(\pi(x)^{-1}-\pi\left(x_{0}\right)^{-1}\right) e_{k}^{\pi}\right\| .\left\|\hat{f}(\pi)^{*} e_{k}^{\pi}\right\| .
\end{aligned}
$$

Now, since $f \in A(G)$ has compact support, then $f \in L^{1}(G)$ and, by Theorem 2.2, $\hat{f}(\pi)=\pi(f) K_{\pi}$. Using the equality $\pi(f)^{*}=\pi\left(\Delta^{-1} \tilde{f}\right)$ and Lemma 2.1,

$$
\left\|\hat{f}(\pi)^{*} e_{k}^{\pi}\right\|=\left\|K_{\pi} \pi(f)^{*} e_{k}^{\pi}\right\|=\left\|K_{\pi} \pi\left(\Delta^{-1} \tilde{f}\right) e_{k}^{\pi}\right\|=\left\|\pi(\tilde{f}) K_{\pi} e_{k}^{\pi}\right\| \leq\|\pi(\tilde{f})\| .\left\|K_{\pi} e_{k}^{\pi}\right\| .
$$

Moreover, since $\pi(\tilde{f})=\int_{G} \pi(x) \tilde{f}(x) d x=\int_{G} \pi\left(x^{-1}\right) \bar{f}(x) \Delta^{-1} x d x$,

$$
\|\pi(\tilde{f})\| \leq \sup _{x \in K} \Delta^{-1}(x) v(K)\|f\|_{\infty} .
$$

Consequently,

$$
\left|f(x)-f\left(x_{0}\right)\right| \leq \sup _{x \in K} \Delta^{-1}(x) v(K)\|f\|_{\infty} \mu(\widehat{H}) \sup _{\pi \in \widehat{H}} \sum_{k=1}^{d_{\pi}}\left\|\left(\pi(x)^{-1}-\pi\left(x_{0}\right)^{-1}\right) e_{k}^{\pi}\right\| .\left\|K_{\pi} e_{k}^{\pi}\right\| \rightarrow 0,
$$


when $x$ tends to $x_{0}$, since $\pi(x)^{-1}$ tends to $\pi\left(x_{0}\right)^{-1}$ strongly (that is, in the strong operator topology). Hence, by the Ascoli-Arzelà theorem, $B_{K, M_{\widehat{H}}}(G)$ is compact. By Riesz's theorem that every locally compact normed space is finite dimensional, it follows that $A_{K, M_{\overparen{H}}}(G)$ is finite dimensional.

Remark 2.5. Let $f \in A(G)$. It is known that $\|f\|_{\infty} \leq\|f\|_{A(G)}$. So, an important consequence of Theorem 2.4 is that the two norms $\|\cdot\|_{\infty}$ and $\|\cdot\|_{A(G)}$ are equivalent on $A_{K, M_{\overparen{H}}}(G)$.

THEOREM 2.6. Suppose that $G$ is connected and noncompact and $f \in A(G)$ has compact support. Then $f=0$ if and only if its regularised Fourier cotransform $\hat{f}$ is compactly supported and $\operatorname{Im} \hat{f}$ is finite dimensional. Thus, the CQUP is valid on $G$.

Proof. Suppose that $f \in A(G)$ has compact support $K$ such that supp $\hat{f} \subseteq \widehat{H}$ and $\operatorname{Im} \hat{f} \subseteq M:=\int_{\widehat{G}}^{\oplus} M_{\pi} d \mu(\pi)$, where $\widehat{H}$ is a compact subset of $\widehat{G}$ and $\operatorname{dim} M \leq d \in \mathbb{N}^{*}$. Towards a contradiction, suppose that $f \neq 0$ and take a compact subset $K_{1}$ of $G$ such that $K \subseteq K_{1}^{\circ}$, the interior of $K_{1}$. By Theorem 2.4, $A_{K_{1}^{\circ}, M_{\widehat{H}}}(G)$ has finite dimension $n$ and

$$
\{0\} \neq A_{K, M_{\overparen{H}}}(G) \subseteq A_{K_{1}^{\circ}, M_{\widehat{H}}}(G) .
$$

Let $\left\{f_{i}: 1 \leq i \leq n\right\}$ be a basis of $A_{K_{1}^{\circ}, M_{\overparen{H}}}(G), K_{2}=\bigcup_{i=1}^{n} \operatorname{supp} f_{i}$ and $N\left(K_{2}\right)$ be an open neighbourhood of $K_{2}$ such that

$$
A_{K_{2}, M_{\overparen{H}}}(G)=A_{N\left(K_{2}\right), M_{\overparen{H}}}(G) .
$$

Let $\varepsilon_{x}, x \in G$, be the function defined on $G$ by $\varepsilon_{x}(t)=0$ if $t \neq x$ and $\varepsilon_{x}(x)=1$. By Theorem 2.2 and Lemma 2.1, for $1 \leq i \leq n$,

$$
\begin{aligned}
\widehat{f_{i} * \varepsilon_{x}}(\pi)=\mathcal{F}\left(f_{i} * \varepsilon_{x}\right)(\pi) K_{\pi} & =\mathcal{F}\left(f_{i}\right)(\pi) \pi\left(\varepsilon_{x}\right) K_{\pi} \\
& =\mathcal{F}\left(f_{i}\right)(\pi) K_{\pi} \pi\left(\Delta^{-1} \varepsilon_{x}\right)=\widehat{f_{i}} \pi\left(\Delta^{-1} \varepsilon_{x}\right) .
\end{aligned}
$$

Thus, $\operatorname{Im}\left({\widehat{f_{i} * \varepsilon_{x}}}_{x}(\pi)\right) \subseteq M_{\pi}$ and $\operatorname{supp}\left(\widehat{f_{i} * \varepsilon_{x}}\right) \subseteq M_{\widehat{H}}$.

Suppose that $x \rightarrow e$, the neutral element of $G$. Then $f_{i} * \varepsilon_{x} \in A_{N\left(K_{2}\right), M_{\bar{H}}}(G)$ for all $i$ and so $f_{i} * \varepsilon_{x} \in A_{K_{2}, M_{\widehat{H}}}(G)$. Consequently, there is an open neighbourhood $N(e)$ of $e$ such that $K_{2} N(e) \subseteq K_{2}$ and thus $K_{2} N(e)=K_{2}$ is open and compact. But this is impossible, since $G$ is connected and noncompact. This contradiction completes the proof.

Remark 2.7. These results generalise their analogues on $G=a x+b$, the affine group of a local field, a particular case of a nonunimodular group (see [12, Theorem 4 and Corollary 5, page 495]). Indeed, if $G=a x+b$, then, for any $f \in A(G)$, supp $\hat{f}=\{\pi\}$ is compact, where $\pi$ is (up to equivalence) the unique infinite-dimensional irreducible unitary representation of $G$ (see [3, page 209]). So, $A_{K, M_{\overparen{H}}}(G)$ reduces to the form $A_{K, M}(G)=\{f \in A(G): \operatorname{supp} f \subseteq K$ and $\operatorname{Im} \hat{f} \subseteq M\}$.

Corollary 2.8. If the CQUP fails on $G$, then $G$ contains an open compact subgroup. 
Proof. If the CQUP fails on $G$, then, by the proof of Theorem 2.6, there exists a nonempty open compact subset $K_{2}$ of $G$. It follows that $K_{2}^{-1} K_{2}$ is a compact neighbourhood of the identity and hence, in view of [4, Theorem 7.5, page 61], $G$ contains an open compact subgroup.

REMARK 2.9. In some unimodular cases, for example, if $G$ is abelian or of bounded representation dimension, that is, $\sup \{\operatorname{dim} \pi: \pi \in \widehat{G}\}<\infty$, then $A_{K, M_{\overparen{H}}}(G)$ reduces to the form $A_{K, \widehat{H}}(G)=\{f \in A(G): \operatorname{supp} f \subseteq K$ and supp $\hat{f} \subseteq \widehat{H}\}$, since $\operatorname{Im} \hat{f}$ is always finite dimensional. In these cases, the CQUP holds on $G$ if and only if

$$
C_{F}(G):=\{f \in A(G): \operatorname{supp} f \text { is compact and supp } \hat{f} \text { is compact }\}=\{0\} .
$$

Corollary 2.10. If $G$ is abelian, then the CQUP holds on $G$ if and only if the identity component of $G$ is noncompact.

Proof. Suppose that $G$ contains an open compact subgroup $H$ and let $1_{H}$ be the characteristic function of $H$. Then $1_{H} \in C_{F}(G)$ and so the converse of Corollary 2.8 is true. It follows that the CQUP holds on $G$ if and only if $G$ does not contain any open compact subgroup, which is in turn equivalent to saying that the identity component of $G$ is noncompact.

Remark 2.11. Suppose that $G$ is abelian and let $f \in L^{1}(G), A_{f}=\{x \in G: f(x) \neq 0\}$ and $B_{f}=\{\hat{x} \in \widehat{G}: \hat{f}(\hat{x}) \neq 0\}$. Following [5], the group $G$ is said to satisfy the QUP if for every $f \in L^{1}(G)$,

$$
v\left(A_{f}\right)<v(G) \quad \text { and } \quad \mu\left(B_{f}\right)<\mu(\widehat{G}) \Rightarrow f=0 \text { a.e. }
$$

If $G$ is abelian and noncompact, then, by [5, Theorem 1, page 137, and Theorem 2, page 139], $G$ satisfies the QUP if and only if the identity component of $G$ is noncompact. Thus, the CQUP and the QUP are equivalent on noncompact abelian groups. However, from [6, page 588], for the definition of the QUP in the (nonabelian) unimodular case, the Plancherel measure $\mu$ is replaced by the measure $\mu^{\prime}$ defined by $\mu^{\prime}(\widehat{E})=\int_{\widehat{E}} \operatorname{dim} \pi d_{\mu}(\pi)$, where $\widehat{E}$ is a $\mu$-measurable subset of $\widehat{G}$. If $G$ is of bounded representation dimension, then the condition $\mu^{\prime}\left(B_{f}\right)<\mu^{\prime}(\widehat{G})$ reduces to the form $\mu\left(B_{f}\right)<\mu(\widehat{G})$ and, by [6, Theorem 2.2, page 590, and Theorem 2.4, page 592], the CQUP and the QUP are harmonically equivalent on such $G$ which are neither compact nor discrete. Note that, in our definition of the CQUP, we retained the Plancherel measure $\mu$ in all cases (abelian, unimodular and nonunimodular).

\section{Acknowledgement}

I would like to express my appreciation to the anonymous referee for valuable comments and suggestions which helped to improve the manuscript. 


\section{References}

[1] D. Arnal and J. Ludwig, 'Q.U.P. and Paley-Wiener properties of unimodular, especially Lie groups', Proc. Amer. Math. Soc. 125(4) (1997), 1071-1080.

[2] M. Duflo and C. C. Moore, 'On the regular representation of a nonunimodular locally compact group', J. Funct. Anal. 21(2) (1976), 209-243.

[3] P. Eymard and M. Terp, 'La transformation de Fourier et son inverse sur le groupe des $a x+b$ d'un corps local', in: Analyse harmonique sur les groupes de Lie (Sém. Nancy-Strasbourg 1976-1978), II, Lecture Notes in Mathematics, 739 (Springer, Berlin, 1979), 207-248.

[4] E. Hewitt and K. A. Ross, Abstract Harmonic Analysis, Vol. I, 2nd edn, Grundlehren der mathematischen Wissenschaften, 115 (Springer, New York, 1979).

[5] J. A. Hogan, 'A qualitative uncertainty principle for locally compact abelian groups', in: Miniconf. Harmonic Analysis and Operator Algebras, Canberra, 1987, Proceedings of the Centre for Mathematical Analysis, Australian National University, Canberra, 16 (1988), 133-142.

[6] J. A. Hogan, 'A qualitative uncertainty principle for unimodular groups of type I', Trans. Amer. Math. Soc. 340 (1993), 587-594.

[7] E. Kaniuth, 'Minimizing functions for an uncertainty principle on locally compact groups of bounded representation dimension', Proc. Amer. Math. Soc. 135 (2007), 217-227.

[8] E. Kaniuth, 'Qualitative uncertainty principles for groups with finite dimensional irreducible representations', J. Funct. Anal. 257 (2009), 340-356.

[9] E. Kaniuth, A. T. Lau and G. Schlichting, 'A topological Paley-Wiener property for locally compact groups', Proc. Amer. Math. Soc. 133(7) (2005), 2157-2164.

[10] G. Kutyniok, 'A weak qualitative uncertainty principle for compact groups', Illinois J. Math. 47 (2003), 709-724.

[11] R. L. Lipsman, 'Non-abelian Fourier analysis', Bull. Sci. Math. (2) 98 (1974), 209-233.

[12] W. Nasserddine, 'Sur le groupe affine d'un corps local', C. R. Math. Acad. Sci. Paris Ser. I 342(7) (2006), 493-495.

[13] W. Nasserddine, 'Une caractérisation de l'algèbre de Fourier pour certains groupes localement compacts', C. R. Math. Acad. Sci. Paris Ser. I 355 (2017), 543-548.

[14] W. Nasserddine, 'Some Fourier analysis results on certain non-abelian groups', submitted.

WASSIM NASSERDDINE, Faculty of Sciences - Section I, Lebanese University, 2905-3901 Hadath, Beirut, Lebanon e-mail: wassim.nasserddine@ul.edu.lb 